\title{
Prediction of Outcome of Acutely Intoxicated Patients Admitted To Intensive Care Unit in Mansoura-Dakhlia Governorate-Egypt
}

\author{
Merfat Oreby , Aisha Maklad', Nihal Shihab² and Hoda Kassem ${ }^{3}$ \\ ${ }^{1}$ Forensic Medicine and Clinical Toxicology Department, Faculty of Medicine, Tanta University, Egypt \\ 2 Public and Community Medicine Department, Faculty of Medicine, Tanta University, Egypt \\ ${ }^{3}$ Emergency Medicine and Traumatology Department, Faculty of Medicine, Tanta University, Egypt
}

\begin{abstract}
ute poisoning is a frequent cause of admission to emergency departments and intensive care units, as it is Isidered as an important medical emergency requiring early diagnosis and rapid initiation of therapy. $\geq$ aim of the present study was to assess characteristics, and outcomes of acutely intoxicated patients who re admitted to ICU. Also, design a scoring system regarding priority for ICU admission. The present dy was carried out on 40 patients who were admitted to intensive care unit of El Mansoura General spitals- Egypt during a period of 6 months. Personal, toxicological, and medical histories, clinical mination and routine investigations were recorded for each patient. At discharge; all patients were ssessed for determination of patients' outcome. The patients were mostly females, single; student aged ; than 30 years and from rural areas. The majority of the patients $(72.5 \%)$ were recovered, $22.5 \%$ were $\mathrm{d}$ and $5 \%$ were referred to hyperbaric oxygen therapy units after carbon monoxide intoxication. Many ients were presented by constricted pupil $(45 \%)$, respiratory depression $(42.5 \%)$, altered mental status $.5 \%)$, tachycardia $(47.5 \%)$, and nausea \& vomiting $(75 \%)$. The highest numbers of cases were poisoned medicinal drugs $(40 \%)$ followed by agricultural chemicals $(35 \%)$. Statistically significant difference ween occupation, toxicological amount $\&$ frequency, pulse rate, Glasgow coma score, $\mathrm{PH} \& \mathrm{PCO}_{2}$ and e of poison (zinc phosphide) and patient outcomes was observed. Although many of the patients' racteristics were significantly associated with patient outcome yet none of them was considered nificant predictors for death. So, the suggested scoring system depended mainly on clinical data and ıple rapid investigation that may be helpful for rapid assessment of the patients after further validation. : recommend the presence of a channel of communication between the general care centers and the son control centers for consultation about the protocol of treatment of poisoned patients. Nationwide, a chanism of communication between the poison centers must be done to benefit from the erent expertise and to make a uniform protocol for treatment of poisoned cases to decrease the mortality $:$ and the burden on hospitals resources.
\end{abstract}

Keywords Intensive care unit; Poison; Outcome; Score; Characteristics

\section{Introduction}

$\mathrm{P}$ oisoning is a common health problem in all countries affecting people in all social levels and age groups. Poisoning events and poisoning-related fatalities is rapidly growing worldwide due to multiple and complex factors such as the wide availability of chemicals and their extensive use for various purposes (in medicine, agriculture, industry.....ect). So, many health threats may occur necessitating hospitalization that put high economic burden on victims and governments and strains on hospital services (Clark, 2004).
In different countries, $0.7 \%$ to $15 \%$ of all emergency department admissions were attributed to acute intoxication.

In Finland, admission to ICU due to poisoning was estimated to range between $3.7-40 \%$ of poison related hospitalization (Liisanantti, 2012).

Intensive care units (ICU) are sections within a hospital that look after patients whose conditions are life-threatening and need constant, close monitoring and support from more technically oriented tools and medication to save life (Takrouri, 2004). 
Patients with severe and life threatening intoxications, requiring intensive treatment and close monitoring in light of the potentially unpredictable clinical courses are usually admitted to the intensive care unit. Acute poisoning due to drugs or chemicals is one of the most common causes leading to emergency department visit, morbidity and mortality (Lam et al., 2010; Kavalci et al., 2012).

One of the principal factors that limit patient's admission to ICU is shortage of ICU beds which costs three times more per day than an acute ward bed as a result of the high levels of investment committed to these specialized centers for the treatment of critical ill patients (Caldeira et al., 2010).

The ICU admission guidelines are intended to encourage effective use of ICU resources without compromising patient care. Current medical literature allows us to develop only very general guidelines. Further clinical studies on the poisoned patient will allow refinement of these guidelines (Chiu et al., 2011)

There is scarcity of data regarding the profile and outcome of patients presenting with acute poisoning and admitted to intensive care units in Egypt.

The aim of the present study was

1-To assess frequency, characteristic, and outcomes of acutely intoxicated patients admitted to intensive care unit of El -Mansoura General Hospital.

2- To suggest a scoring system to provide helpful data as regards priority for ICU admission.

\section{Patients and methods}

\section{Patients}

This is a prospective study that was carried out on all patients admitted to ICU of El- Mansoura General Hospital (Belonging to the Ministry Health) with alleged acute poisoning intake or drugs overdose during a period of six months from the start of November 2013 to the end of April 2014. All acutely intoxicated patients that were admitted to the intensive care unit during the study period were included. Only patients with chronic intoxication were excluded.

Written consent was obtained from every patient or his legal relative after complete explanation of the nature and the purpose of the study. Confidentiality of the patients' records was maintained. This study was approved by the Ethical Committee of the Faculty of Medicine, Tanta University.

\section{Methods}

I- At admission: all patients were subjected to:

1. Detailed history taking including the following:

a. Personal history: included name, age, sex, marital status, residence, occupation and special habits.

b. Toxicological history: included type of poison, (based mainly on history, clinical examination and sometimes investigation) amount, route of exposure, frequency (single or repeated within 8 hours), period of exposure and time passed since administration, place, circumstance of poisoning, method of transfer, prehospital consultation and method of gastrointestinal decontamination.

c. Past history: included attempts of suicide and any history of chronic disease or psychic trouble.

\section{General and clinical examination of the} following:

-Vital signs (pulse, blood pressure, temperature and respiratory rate), pupillary state and mental status were assessed by Glasgow coma scale.

-Neurological, respiratory, cardiovascular and GIT systems examinations.

\section{Laboratory investigations:}

Serum electrolytes, arterial blood gases, complete blood count, blood glucose level, liver function (alanine aminotransferase and albumin) and renal function tests (urea level) were done.

4. ECG.

II- At discharge: All patients were reassessed for determination of patient's prognosis and outcome by either recovery or death.

\section{Statistical analysis}

Statistical presentation and analysis of the present study was conducted, using SPSS Version 16. The Student t-test, Mann Whitney test and Paired t test, were used for the quantitative data and chi-square test, fisher exact test or Monte Carlo exact test for qualitative data. Pearson correlation coefficient test and Spearman correlation coefficient test were used for quantifying the relationship between two variables. Logistic Regression analysis was used to analyze the predictor parameters for patient's outcome and prognosis. $\mathrm{P}$ value was considered significant if $<0.05$.

\section{Results}

The total cases that were admitted to El- Mansoura General Hospital during the period of the study were 7200 , out of them; $384(5.33 \%)$ were admitted to I.C.U. The total poisoned cases were 1300 constituting 18.06 $\%$ of the total hospital admissions, of which 40(3.08\%) cases were admitted to I.C.U. The forty patients of the present study represented $10.42 \%$ of the total patients that were admitted to the ICU and $0.56 \%$ of total hospital admission cases.

Table (1) demonstrated that the major ICU admission criteria were hemodynamic instability (47.5\%) (Physical signs of acute circulatory failure constitute primary references for shock, include hypotension, abnormal heart rate, cold extremities, peripheral cyanosis together with bedside measurements of right-sided filling pressure and decreased urine flow.) or altered mental status (42.5\%) or combined hemodynamic instability and altered mental status (35\%). The majority of the admitted patients to ICU $(72.5 \%)$ were recovered and $22.5 \%$ died. Only two patients $(5 \%)$ were poisoned by carbon mono oxide and were referred to hyperbaric oxygen therapy units.

The patients of the current study were mostly females (55\%), single (57.5), student (47.5) from rural areas $(60 \%)$, and aged less than 30 years $(67.5 \%)$. The relationship between socio-demographic characteristics and patient outcomes revealed statistically significant difference regarding occupation (Table 2).

Table (3) demonstrated that, 35\%, 27.5\% and $12.5 \%$ of the studied patients were suffering from psychic troubles, medical or surgical diseases and 
suicidal attempts respectively. Additionally, the most common route of intoxication was ingestion $(72.5 \%)$ and the least route was injection $(5 \%)$. The majority of the patients took the poison in small amount $(85 \%) \&$ once $(87.5 \%)$, at home $(85 \%)$, with a delay time of 1 $24 \mathrm{hrs}$ since poison administration $(95 \%)$, in a suicidal and accidental mode $(52.5 \%$ and $47.5 \%)$ and were transferred by ambulance $(82.5 \%)$ with a pre hospital consultation treatment $(72.5 \%)$. Decontamination (Gastric lavage and activated charcoal administration) were done to $72.5 \%$ of the studied patients. Statistically significant difference was observed between toxicological amount \& frequency and patients' outcome.

Table (4) revealed statistically significant difference regarding cardiovascular system and patient outcomes. The highest percentage of patients were presented by constricted pupil (45\%), respiratory depression $(42.5 \%)$, tachycardia $(47.5 \%)$, convulsion $(17.5 \%)$, and nausea \& vomiting $(75 \%)$. The mean value of Glasgow coma was $8.90 \pm 2.43$.

Statistically significant difference between pulse rate, GCS, $\mathrm{PH}$ and $\mathrm{PCO}_{2}$ and patients outcomes were present in Table (5).

Relationship between types of poison at admission with patient outcomes revealed statistically significant difference regarding zinc phosphid. The highest numbers of admitted poisoned cases were medicinal drugs $(40 \%)$ \{ Tricyclic antidepressant $(12.5 \%)$, tramadol(10\%), benzodiazepines $(7.5 \%)$, and both carbamazepines and morphine $(5 \%)\}$ followed by agricultural chemicals (35\%) \{organophosphorus $(22.5 \%)$, zic phosphide (10\%) and hydrogen cyanamide (2.5)\} and carbon mono oxide poisoned cases (20\%). However, cannabis and botulism poisoned patients accounted for the least percentage (2.5\%) of the admitted poisoned patients to ICU (Table 6).

Table (7) revealed insignificant difference between the length of stay and patient outcome.

Table (8) illustrated the previously mentioned significant patient parameters as predictors for the outcome of death. Neither of the Tested patient characteristics have an effect on the patient outcome as predictors (Non -significant association, $\mathrm{P}>5 \%$ ). As regards occupation, being a student had high odds ratio Exp (B) equal to 230.134 times more likely to outcome of death than the reference employer group. Concerning the history of toxicity, the odds of repeated toxicity was higher than odds for large amount of toxin more likely to the outcome of death $(7.700 \& 1.35$ respectively). Concerning the large amount, there was 1.35 odds to outcome of death more than the reference group (small amount). Regarding cardiovascular manifestations, tachycardia had higher odds than bradycardia $(\mathrm{EXB}=1.140$ \& 0.667 respectively) to outcome of death compared with the reference normal group. As regards pulse rate, each increase in one pulse rate, has a $16.1 \%$ increase of odds of having outcome of death $(\operatorname{Exp}(\mathrm{B})=1.161)$. Regarding PCO2, each increase by one unit, has a $33.2 \%$ increase of odds of having death $(\mathrm{EXB}=1.332)$. As regards both of The $\mathrm{PH}$ and Glasgow coma, $\operatorname{Exp}(\mathrm{B})=0$ means a large difference but with a non-significant effect on the patient outcome of death. Table (9) illustrated the suggested scoring system for patient prognosis. Parameters used in the scoring system were clinical parameters that was supposed to be associated with patient outcome and prognosis (Blood pressure, pulse rate, respiratory rate, Glasgow coma score, convulsions, $\mathrm{PH}, \mathrm{pco}_{2}, \mathrm{po}_{2}, \mathrm{HCo}_{3}, \mathrm{So}_{2} \%, \mathrm{Na}$ and $\mathrm{K}$ levels) bases on clinical experience. They were given scores ranged from 1 to 3 according to their association with the prognosis. The worse prognostic values were given score 3 , and the least score 1 was given to the normal values for those parameters. The normal ranges for all parameters were based on the local laboratory standards. The moderate and severe ranges were based on a clinical perspective and facts in the emergency room.

\section{Details of the suggested scoring system}

As regards the clinical parameters, the suggested scoring system was as follow:

- $\quad$ Score 1 was given for normal values and absence of abnormality.

- Score 2 for the mild prognostic values of the parameters

- Score 3 for the severe prognostic values (serious outcome) for the parameter.

The total score for all the parameters ranged from 12 to 36. The total score is classified as follows:

- Good outcome and prognosis if the score was $=12$ up to $20(\leq 33.3 \%$ of the total score)

- Bad prognosis if the score ranged from 21 to 36 (> $33.3 \%$ of the total score).

So, for each patient in the emergency room, after assessment of all parameters, the total score will be summed and the patient can be classified in either of the two groups mentioned above.

Table (1): Admission criteria of acutely intoxicated patients in ICU (no=40)

\begin{tabular}{|l|l|l|}
\hline Admission criteria & Number & $\%$ \\
\hline Hemodynamic instability & 18 & 47.5 \\
\hline Altered mental status & 17 & 42.5 \\
\hline Hemodynamic instability and altered mental status & 14 & 35 \\
\hline Convulsions & 7 & 17.5 \\
\hline Hemodynamic instability and convulsions & 2 & 5 \\
\hline $\begin{array}{l}\text { Altered mental status } \\
\text { and convulsions }\end{array}$ & 1 & 2.5 \\
\hline
\end{tabular}


Table (2): Socio-demographic characteristics and their relation to patient outcome (no=40)

\begin{tabular}{|c|c|c|c|c|c|}
\hline \multicolumn{3}{|c|}{ Personal history } & Patient & & \multirow{3}{*}{$\begin{array}{l}\text { Fisher's } \\
\text { test }\end{array}$} \\
\hline & & $\begin{array}{l}\text { Recovery and referral } \\
(31)\end{array}$ & Died (9) & Total (40) & \\
\hline & & Number $(\%)$ & Number (\%) & Number $(\%)$ & \\
\hline \multirow{2}{*}{ Age } & $<30 y$ years & $20(81.1)$ & $7(18.9)$ & $27(67.5)$ & \\
\hline & $\geq 30$ years & $11(84.6)$ & $2(15.4)$ & $13(32.5)$ & \\
\hline \multirow{2}{*}{ Sex } & Male & $16(88.9)$ & $2(11.1)$ & $18(45)$ & \multirow{2}{*}{0.15} \\
\hline & Female & $15(68.2)$ & $7(31.8)$ & $22(55)$ & \\
\hline \multirow{2}{*}{ Residence } & Urban & $14(87.5)$ & $2(12.5)$ & $16(40)$ & \multirow{2}{*}{0.27} \\
\hline & Rural & $17(70.8)$ & $7(29.2)$ & $24(60)$ & \\
\hline \multirow{3}{*}{$\begin{array}{l}\text { Marital } \\
\text { status }\end{array}$} & Married & $10(76.9)$ & $3(23.1)$ & $13(32.5)$ & \multirow{3}{*}{1.0} \\
\hline & Single & $18(78.3)$ & $5(21.7)$ & $23(57.5)$ & \\
\hline & $\begin{array}{l}\text { Divorced } \quad \& \\
\text { widow }\end{array}$ & $3(75.0)$ & $1(25.0)$ & $4(10)$ & \\
\hline \multirow{4}{*}{ Occupation } & Student & $15(78.9)$ & $4(21.1$ & $19(47.5)$ & \multirow{4}{*}{$0.038 *$} \\
\hline & Employed & $8(100.0)$ & $0(0.0)$ & $8(20)$ & \\
\hline & Unemployed & $4(44.4)$ & $5(55.6)$ & $9(22.5)$ & \\
\hline & Worker & $4(100.0)$ & $0(0.0)$ & $4(10)$ & \\
\hline
\end{tabular}

$* P$ value is significant if $<0.05$

Table (3): Patient habits, past \& toxicological history and their relation to patient outcome (no=40)

\begin{tabular}{|c|c|c|c|c|c|c|}
\hline \multirow{3}{*}{ History } & & & \multicolumn{2}{|c|}{ Patient outcome } & \multirow[b]{2}{*}{$\begin{array}{c}\text { Total } \\
(40)\end{array}$} & \multirow{3}{*}{$\begin{array}{l}\text { Fisher's } \\
\text { exact test }\end{array}$} \\
\hline & & & \multirow{2}{*}{$\begin{array}{c}\begin{array}{c}\text { Recovery and } \\
\text { referral }(31)\end{array} \\
\text { Number }(\%)\end{array}$} & \multirow{2}{*}{$\begin{array}{c}\begin{array}{c}\text { Died } \\
(9)\end{array} \\
\begin{array}{c}\text { Number } \\
(\%)\end{array}\end{array}$} & & \\
\hline & & & & & $\begin{array}{c}\text { Number } \\
(\%)\end{array}$ & \\
\hline \multirow{3}{*}{$\begin{array}{l}\text { Patient } \\
\text { habits }\end{array}$} & & Smoker & $10(100)$ & $0(0.0)$ & $10(25)$ & \multirow{3}{*}{0.36} \\
\hline & & Addict & $5(83.3)$ & $1(16.7)$ & $6(15)$ & \\
\hline & & Non & $16(66.7)$ & $8(33.3)$ & $24(60)$ & \\
\hline \multirow[t]{4}{*}{ Past history } & & suicide & $3(60.0)$ & $2(40.0)$ & $5(12.5)$ & \multirow{4}{*}{0.41} \\
\hline & & disease & $10(90.9)$ & $1(9.1)$ & $11(27.5)$ & \\
\hline & & $\begin{array}{l}\text { psychic } \\
\text { trouble }\end{array}$ & 11(78.6) & $3(21.4)$ & $14(35)$ & \\
\hline & & Non & $7(70.0)$ & $3(30.0)$ & $10(25)$ & \\
\hline \multirow{17}{*}{$\begin{array}{l}\text { Toxicological } \\
\text { history }\end{array}$} & \multirow{3}{*}{ Route } & Ingestion & $23(79.3)$ & $6(20.7)$ & $29(72.5)$ & \multirow{3}{*}{0.51} \\
\hline & & Injection & $1(50.0)$ & $1(50.0)$ & $2(5)$ & \\
\hline & & Inhalation & $7(77.8)$ & $2(22.2)$ & $9(22.5)$ & \\
\hline & \multirow{2}{*}{$\begin{array}{l}\text { Circumstances of } \\
\text { poisoning }\end{array}$} & Accidental & $16(84.2)$ & $3(15.8)$ & $19(47.5)$ & \multirow{2}{*}{0.46} \\
\hline & & Suicidal & $15(71.4)$ & $6(28.6)$ & $21(52.5)$ & \\
\hline & \multirow{2}{*}{ amount } & small & $30(88.2)$ & $4(11.8)$ & $34(85)$ & \multirow{2}{*}{$0.00 *$} \\
\hline & & large & $1(16.7)($ & $5(83.3)$ & $6(15)$ & \\
\hline & \multirow{2}{*}{$\begin{array}{l}\text { Frequency of intake } \\
\text { within } 8 \text { hrs }\end{array}$} & Once & $30(85.7)$ & $5(14.3)$ & $35(87.5)$ & \multirow{2}{*}{$0.01 *$} \\
\hline & & repeated & $1(20)$ & $4(80)$ & $5(12.5)$ & \\
\hline & \multirow{2}{*}{ Transfer } & Ambulance & $25(75.8)$ & $8(24.2)$ & $33(82.5)$ & \multirow{2}{*}{1.0} \\
\hline & & Family & $6(85.7)$ & $1(14.3)$ & $7(17.5)$ & \\
\hline & \multirow{2}{*}{$\begin{array}{c}\text { Time since } \\
\text { administration }\end{array}$} & 1-24hrs & $31(81.6)$ & $7(18.4)$ & $38(95)$ & \multirow[b]{2}{*}{0.12} \\
\hline & & $>24 \mathrm{hrs}$ & 0 & $2(100)$ & $2(5)$ & \\
\hline & \multirow[t]{2}{*}{ Place } & Home & $27(79.4)$ & $7(20.6)$ & $34(85)$ & \multirow{2}{*}{0.51} \\
\hline & & Outside & $4(66.7)$ & $2(33.3)$ & $6(15)$ & \\
\hline & \multirow{2}{*}{$\begin{array}{l}\text { Gastrointestinal } \\
\text { decontamination }\end{array}$} & Yes & $23(79.3)$ & $6(20.7)$ & $29(72.5)$ & \\
\hline & & No & $8(72.7)$ & $3(27.3)$ & $11(27.5)$ & \\
\hline
\end{tabular}

* P value is significant if $<0.05$ 
Table (4): Clinical presentation at admission and their relation to patient outcomes $($ no=40)

\begin{tabular}{|c|c|c|c|c|c|}
\hline \multirow{2}{*}{\multicolumn{2}{|c|}{$\begin{array}{r}\text { Clinical presentation at } \\
\text { Admission }\end{array}$}} & \multicolumn{2}{|l|}{ Patient outcome } & \multirow[b]{2}{*}{$\begin{array}{l}\text { Total } \\
(40)\end{array}$} & \multirow{3}{*}{$\begin{array}{l}\text { Fishr's } \\
\text { exact }\end{array}$} \\
\hline & & \multirow{2}{*}{\begin{tabular}{|l|}
$\begin{array}{l}\text { Recovery and } \\
\text { referral (31) }\end{array}$ \\
Number (\%) \\
\end{tabular}} & \multirow{2}{*}{$\begin{array}{l}\begin{array}{l}\text { Died } \\
(9)\end{array} \\
\text { Number }(\%) \\
\end{array}$} & & \\
\hline & & & & Number (\%) & \\
\hline Convulsions & Yes & $7(100.0)$ & $0(0.0)$ & $7(17.5)$ & \multirow{2}{*}{0.17} \\
\hline & No & $24(72.7)$ & $9(27.3)$ & $33(82.5)$ & \\
\hline \multirow{3}{*}{ Pupil reaction } & Normal & $9(64.3)$ & $5(35.7)$ & $14(35)$ & \multirow{3}{*}{0.16} \\
\hline & Constricted & $14(77.8)$ & $4(22.2)$ & $18(45)$ & \\
\hline & Dilated & $8(100.0)$ & $0(0.0)$ & $8(20)$ & \\
\hline \multirow{4}{*}{$\begin{array}{l}\text { Respiratory } \\
\text { System }\end{array}$} & Normal & $4(100.0)$ & $0(0.0)$ & $4(10)$ & \multirow{4}{*}{0.77} \\
\hline & Crepitation & $9(75.0)$ & $3(25.0)$ & $12(30)$ & \\
\hline & $\begin{array}{l}\text { Respiratory } \\
\text { distress }\end{array}$ & $6(85.7)$ & $1(14.3)$ & $7(17.5)$ & \\
\hline & $\begin{array}{l}\text { Respiratory } \\
\text { depression }\end{array}$ & $12(70.6)$ & $5(29.4)$ & $17(42.5)$ & \\
\hline \multirow{3}{*}{$\begin{array}{l}\text { Cardiovascular } \\
\text { System }\end{array}$} & Normal & $3(100.0)$ & $0(0.0)$ & $3(7.5)$ & \multirow{3}{*}{$0.023^{*}$} \\
\hline & Tachycardia & $11(57.9)$ & $8(42.1)$ & $19(47.5)$ & \\
\hline & Bradycardia & $17(94.4)$ & $1(5.6)$ & $18(45)$ & \\
\hline \multirow[b]{2}{*}{ Gastrointestinal system } & Normal & $9(90.0)$ & $1(10.0)$ & $10(25)$ & \multirow[b]{2}{*}{0.40} \\
\hline & $\begin{array}{l}\text { Nausea and } \\
\text { vomiting }\end{array}$ & $22(73.3)$ & $8(26.7)$ & $30(75)$ & \\
\hline
\end{tabular}

$* P$ value is significant if $<0.05$

Table (5): Relationship between patient's parameters at admission with their outcome (no=40)

\begin{tabular}{|c|c|c|c|c|c|c|}
\hline \multirow{3}{*}{$\begin{array}{l}\text { Basic parameters } \\
\text { At admission }\end{array}$} & \multicolumn{4}{|c|}{ Patient outcome } & \multirow{3}{*}{\begin{tabular}{l}
\multicolumn{2}{c}{ t test } \\
or Mann- \\
Whitney
\end{tabular}} & \multirow{3}{*}{$P$ value } \\
\hline & \multicolumn{2}{|c|}{$\begin{array}{l}\text { Recovery and referral } \\
\text { (31) }\end{array}$} & \multicolumn{2}{|l|}{ Died (9) } & & \\
\hline & $\begin{array}{l}\text { Mean } \\
\text { Or } \\
\text { median }\end{array}$ & $\begin{array}{l}\text { Std. } \\
\text { Deviation } \\
\text { or Range }\end{array}$ & $\begin{array}{l}\text { Mean } \\
\text { Or } \\
\text { median }\end{array}$ & $\begin{array}{l}\text { Std. } \\
\text { Deviation } \\
\text { Or range }\end{array}$ & & \\
\hline Age & 26.97 & 19.177 & 19.33 & 10.817 & 1.136 & 0.263 \\
\hline Systolic blood pressure & 103.23 & 17.961 & 103.23 & 22.095 & 1.537 & 0.133 \\
\hline Diastolic blood pressure & 68.87 & 14.184 & 58.89 & 14.743 & 1.843 & 0.073 \\
\hline Pulse rate & 88.55 & 23.352 & 108.11 & 24.091 & 2.198 & $0.034 *$ \\
\hline Respiratory rate\# & 12.00 & 2.00 & 12.00 & 2.00 & $\mathrm{Z}=0.912$ & 0.362 \\
\hline Glasgow coma score & 9.77 & 1.910 & 5.89 & 1.364 & 5.673 & $0.000^{*}$ \\
\hline PH(Power of hydrogen) & 7.340 & 0.100 & 7.138 & 0.149 & 4.742 & $0.000 *$ \\
\hline $\begin{array}{l}\mathrm{PCO}_{2} \text { (Partial carbon dioxide } \\
\text { Pressure) }\end{array}$ & 34.94 & 11.588 & 52.52 & 25.050 & 3.010 & $0.005 *$ \\
\hline $\mathbf{P O}_{2} \#($ Partialoxygen pressure) & 61.900 & 66.70 & 96.80 & 168.70 & $\mathrm{Z}=0.778$ & 0.437 \\
\hline HCO3(Blood bicarbonate) & 20.187 & 2.329 & 21.056 & 5.025 & 0.740 & 0.464 \\
\hline $\mathbf{N a}($ Serum sodium) & 143.69 & 9.549 & 146.111 & 13.513 & 0.610 & 0.545 \\
\hline $\mathbf{K}($ Serum potassium) & 3.18 & 0.806 & 3.68 & 0.792 & 0.795 & 0.112 \\
\hline WBC(White blood cell ) & 12.09 & 4.259 & 13.84 & 4.203 & 1.089 & 0.283 \\
\hline RBC(Red blood cell) & 4.449 & 0.681 & 4.480 & 1.049 & 0.084 & 0.935 \\
\hline Platelets count & 273.13 & 64.557 & 257.00 & 98.948 & 0.461 & 0.654 \\
\hline HGB(Hemoglobin ) & 11.67 & 1.181 & 10.89 & 1.867 & 1.525 & 0.136 \\
\hline $\begin{array}{l}=\mathbf{A L T} \quad \# \quad \text { (Alanine amino } \\
\text { transferase) }\end{array}$ & 30.00 & 12.00 & 31.00 & 4.00 & $Z=-1.258$ & 0.209 \\
\hline ALB( Serum albumin) & 4.090 & 0.4066 & 3.830 & 0.9369 & 0.812 & 0.438 \\
\hline glucose Blood & 122.48 & 36.578 & 121.56 & 13.866 & 0.074 & 0.941 \\
\hline Serum creatinine & 0.910 & 0.320 & 0.833 & 0.312 & 0.634 & 0.530 \\
\hline $\begin{array}{l}\text { SO2\# (Arterial oxygen } \\
\text { saturation) }\end{array}$ & 85.00 & 13.00 & 85.00 & 12.85 & $\mathrm{Z}=0.18$ & 0.857 \\
\hline
\end{tabular}

\#= non parametric data (median, range and Mann Whitney test were calculated in non-parametric data)

$$
* P \text { value is significant if }<0.05 \quad \text { T test: student } t \text { test } \quad Z \text { test: Mann-Whitney test. }
$$


Table (6): Toxicological diagnoses at admission and their relation to patient outcome (no=40)

\begin{tabular}{|l|l|l|l|l|}
\hline \multirow{2}{*}{ Diagnosis } & \multicolumn{2}{|c|}{ Patient outcome } & Total & \\
& \multicolumn{2}{|c|}{$\begin{array}{l}\text { Recovery and } \\
\text { referral (31) }\end{array}$} & $\begin{array}{l}\text { Died } \\
(9)\end{array}$ & $\begin{array}{l}\text { Fisher's } \\
\text { exact }\end{array}$ \\
\hline & Number (\%) & Number (\%) & $\begin{array}{l}\text { Number } \\
(\%)\end{array}$ & \\
\hline Organophosphorous & $6(66.7)$ & $3(33.3)$ & $9(22.5)$ & 0.65 \\
\hline Carbon monoxide & $6(75.0)$ & $2(25.0)$ & $8(20)$ & 1 \\
\hline Tricyclicantidepresant & $5(100.0)$ & $0(0.0)$ & $5(12.5)$ & 0.32 \\
\hline Zinc phosphida & $1(25.0)$ & $3(75.0)$ & $4(10)$ & $0.03^{*}$ \\
\hline Tramdol & $4(100.0)$ & $0(0.0)$ & $4(10)$ & 0.56 \\
\hline Benzodiazepine & $3(100.0)$ & $0(0.0)$ & $3(7.5)$ & 0.58 \\
\hline Carbamazepine & $2(100.0)$ & 0.0 & $2(5)$ & 1 \\
\hline Morphine & $1(50.0)$ & $1(50.0)$ & $2(5)$ & 0.41 \\
\hline Hydrogen Cyanamide & $1(100.0)$ & $0(0.0)$ & $1(2.5)$ & 1 \\
\hline Botulism & $1(100.0)$ & $0(0.0)$ & $1(2.5)$ & 1 \\
\hline Cannabis & $1(100.0)$ & $0(0.0)$ & $1(2.5)$ & 1 \\
\hline $\begin{array}{l}\text { Test of significance within } \\
\text { all groups }\end{array}$ & Montcarlo test, p=0.23 & & & \\
\hline
\end{tabular}

$* P$ value is significant if $<0.05$

Table (7): Length of stay in the studied patients in relation to their outcome

\begin{tabular}{|c|c|c|c|c|}
\hline \multirow[b]{2}{*}{ Length of stay } & \multicolumn{2}{|l|}{ Patient outcome } & \multirow{2}{*}{$\begin{array}{l}\text { Mann } \\
\text { Whitney test }\end{array}$} & \multirow[b]{2}{*}{ P value } \\
\hline & $\begin{array}{l}\text { Recovery and } \\
\text { referral }\end{array}$ & Died & & \\
\hline Median & 5 days & 2 days & \multirow{3}{*}{$Z=0.84$} & \multirow{3}{*}{0.24} \\
\hline IQR & 4 days & 13 days & & \\
\hline Range & 1day - 25days & 1 day -33 days & & \\
\hline
\end{tabular}

$I Q R$-inter quartile range

Table (8): Regression analysis for the significant personal and medical characteristics as predictors for the Patient outcome (no=40)

\begin{tabular}{|c|c|c|c|c|}
\hline \multirow{2}{*}{$\begin{array}{l}\text { The significant patient } \\
\text { parameters }\end{array}$} & \multirow[t]{2}{*}{ Sig. } & \multirow[t]{2}{*}{$\operatorname{Exp}(B)$} & \multicolumn{2}{|c|}{ 95.0\% C.I. for $\operatorname{EXP}(B)$} \\
\hline & & & Lower & Upper \\
\hline Occupation (employer) & 1.000 & & & \\
\hline Worker & 1.000 & $1.336 \mathrm{E} 3$ & 0.000 & . \\
\hline Un-employer & 1.000 & 2.639 & 0.000 & . \\
\hline Student & 1.000 & 230.134 & 0.000 & . \\
\hline Cardiovascular (normal) & 1.000 & & & \\
\hline Tachycardia & 0.999 & $1.140 \mathrm{E} 10$ & 0.000 & . \\
\hline Bradycardia & 1.000 & 0.667 & 0.000 & . \\
\hline Toxin large Amount & 1.000 & 1.349E5 & 0.000 & \\
\hline $\begin{array}{l}\text { Toxins taken repeatedly } \\
\text { within 8hrs }\end{array}$ & 1.000 & $7.700 \mathrm{E} 6$ & 0.000 & \\
\hline Pulse rate & 1.000 & 1.161 & 0.000 & \\
\hline PH & 1.000 & 0.000 & 0.000 & . \\
\hline $\mathrm{PCO}_{2}$ & 0.999 & 1.332 & 0.000 & . \\
\hline Glasgow Coma Scale & 0.996 & 0.000 & 0.000 & . \\
\hline Constant & 1.000 & $1.578 \mathrm{E} 152$ & & \\
\hline
\end{tabular}

$* P$ value is significant if $<0.05$ 
Table (9): Suggested scoring system regarding priority for admission to ICU

\begin{tabular}{|c|c|c|c|}
\hline \multirow{2}{*}{$\begin{array}{c}\text { Clinical and laboratory } \\
\text { parameters }\end{array}$} & \multicolumn{3}{|c|}{ Scoring } \\
\hline & $\begin{array}{c}\text { Normal range } \\
\text { (1) }\end{array}$ & $\begin{array}{l}\text { Moderate } \\
\text { (2) }\end{array}$ & $\begin{array}{l}\text { Severe } \\
\text { (3) }\end{array}$ \\
\hline B.P (Blood pressure) & $120 / 80$ & $>140 / 90$ & $<80 / 50$ \\
\hline Pulse rate & $60-100$ & $>100$ & $<60$ \\
\hline R.R (Respiratory rate) & $16-20$ & $>20$ & $<16$ \\
\hline GCS (Glasgow coma scale) & $13-15$ & $9-12$ & $3-8$ \\
\hline Convulsions & No convulsions & convulsions & $\begin{array}{c}\text { Repeated } \\
\text { convulsions }\end{array}$ \\
\hline $\mathbf{P H}($ Power of hydrogen) & $7.35-7.45$ & $>7.45$ & $<7.35$ \\
\hline $\begin{array}{l}\mathbf{P C O}_{2} \text { (Partial carbon dioxide } \\
\text { pressure) }\end{array}$ & $35-45$ & $<35$ & $>45$ \\
\hline $\begin{array}{c}\mathbf{P O}_{2} \\
\text { (Partial oxygen pressure) }\end{array}$ & $100-80$ & $60-80$ & $<60$ \\
\hline $\mathrm{HCO}_{3}$ (Blood bicarbonate) & $22-26$ & $>26$ & $<22$ \\
\hline $\mathbf{S O}_{2} \%$ ( Arterial oxygen saturation) & $92-100$ & $80-92$ & $<80$ \\
\hline Na (Serum sodium) & $135-148$ & $<135$ & $>148$ \\
\hline $\mathbf{K}$ (Serum potassium) & $3.5-4.5$ & $<3.5$ & $>4.5$ \\
\hline
\end{tabular}

\section{Discussion}

Acute poisoning is a frequent cause for admission to emergency departments and one of the most important reasons of hospitalization in intensive care units. It is considered as an important medical emergency requiring early diagnosis and rapid initiation of appropriate therapy (Duger et al., 2013).

The total poisoned cases were 1300 cases representing $18.06 \%$ of total hospital admissions. The forty cases of the present study represented $10.42 \%$, $3.08 \%$ and $0.56 \%$ of total I.C.U, poison center and hospital admission. This partially coincided with the study of Azab (2009) as the total poisoned cases that were admitted to El-Menoufiya Poison Control Center (El-Menoufiya governorate - Egypt) were $1.4 \%$ of total hospital admission, of which $20.2 \%$ have been admitted to ICU and they represent $7.08 \%$ of total ICU admission. However, in Iran (Baharloo Hospital, Tehran.), life-threatening poisoned patients requiring ICU admission constituted $8 \%$ of the total poisoned cases admitted during one year (Taghaddosinejad et al., 2012). Arrkan et al. (2014) found that acute intoxications constituted $7.61 \%$ of the admitted patients to ICU of Karabük Training and Research Hospital in Turkey.

The patients of the current study were mostly females $(55 \%)$, single (57.5), student (47.5), from rural areas $(60 \%)$ and aged less than 30 years $(67.5 \%)$. This is comparable with the findings of previous studies; Singh et al. (2011) found that $50.7 \%$ of the studied cases were males and from age group 21 to 30 years in a study done in a tertiary care hospital in the metropolitan city of New Delhi. Taghaddosinejad et al. (2012) showed that the majority of the studied patients were young (below 30 years); males $(57 \%)$ and either employed (40.2\%), students or housewives.

The present study revealed that $35 \%$ of the patients were suffering from psychic trouble, $27.5 \%$ from medical or surgical diseases and $12.5 \%$ had suicidal attempts. The percentage of patients with previous suicidal attempts or preexisting psychiatric diseases was lower than other studies done in Hong Kong and India (lam et al., 2010; Singh et al., 2011).

Suicidal poisoning in the current study represents $52.5 \%$ of the studied cases. Similarly, Arıkan et al. (2014) stated that suicidal attempt was present in $51.2 \%$ of the studied patients. Manjula et al. (2013) revealed that the most common mode of poisoning in the ICU of a tertiary care hospital in the city of Bangalore-India was suicidal $(86.2 \%)$ followed by accidental $(13.8 \%)$. This high percentage may be attributed to many factors such as poverty, financial \& psychological problems; unemployment or marital conflicts. Moreover, many pressures and stressful conditions as failure in love and exams especially among students may contribute to suicide.

The current study revealed that the most common route of intoxication was ingestion $(72.5 \%)$ followed by inhalation $(22.5 \%)$. In agreement with this, Singh et al. (2011) stated that the poison was taken mainly by ingestion $(97.8 \%)$ followed by intravenous $(1.4 \%)$ then the skin $(0.7 \%)$. Moreover, Manjula et al. (2013) reported that poisoning was oral in all cases. This difference may be explained by the geographic nature of the region, circumstances of poisoning and by the difference in the nature of poisons included in their study and the present study as the route of intake differs according to the nature of poison. Furthermore, the current results revealed that, the highest numbers of admitted cases were intoxicated by organophosphates $(22.5 \%)$ which are available as liquid form that could be taken orally. Additionally; tricyclic antidepressant $(12.5 \%)$, zinc phosphida (10\%) and tramdole $(10 \%)$ are available in oral forms that could be taken by ingestion.

In the present study, the patients were poisoned mainly at home $(85 \%)$, this reflects the 
danger of household products as a cause of acute poisoning. Accordingly, Bronstein et al. (2008) found in the Annual Report of the American Association of Poison Control Centers that the majority of poisoned patients $(92.9 \%)$ were inside the houses.

The majority of the present studied cases had prehospital consultation treatment and decontamination (gastric lavage \& activated charcoal) were done to $72.5 \%$ of the studied patients. In contrast to the present study, Azab (2009) reported that, $41.1 \%$ of the studied patients received pre hospital treatment and gastric lavage was done to $42 \%$ of the studied cases. Taghaddosinejad et al. (2012) found that gastric lavage was done to $88 \%$ of admitted patients to ICU. The present finding is strange as all patients came after one hour of poison administration. As a matter of fact, routine gastric lavage is not recommended in poisoned patient and activated charcoal should be administered to patients presenting with early life threatening poisoning (Vale and Kulig, 2004; Chyka et al., 2005).

In the present study, most of the patients were transferred by ambulance $(82.5 \%)$. This coincides with the results obtained by Azab (2009) in which $64 \%$ of the patients were transferred by ambulance. This may be explained by the severity of cases as most of patients had to be transported from far off villages as $60 \%$ of the studied patients were from rural areas where facilities for managing cases of acute poisoning in terms of qualified medical staff and equipment were either insufficient or nonexistent.

The time passed since poison administration was $1-24 \mathrm{hrs}$ in $95 \%$ of cases. Singh et al., (2011) found that $22.5 \%$ of the patients in their study arrived hospital in more than 6 hours. The longer time passed since poison administration could be attributed to the fact that only two poison control centers are available in Dakahlia Governorate and they are present in the capital of the governorate, although, the majority of the poisoned patients come from rural areas. Additionally, only two intensive care units unspecialized to poisoned cases are present (one belong to the ministry of health and the other to faculty of medicine).

The highest percentages of patients in the present study were presented by constricted pupil (45\%), respiratory depression $(42.5 \%)$, tachycardia $(47.5 \%)$ and nausea \& vomiting $(75 \%)$. The mean value of Glasgow coma was $(8.90 \pm 2.426)$. These findings are in agreement with Azab (2009) who revealed that most of the studied cases were presented with coma, vomiting and constricted pupils. Taghaddosinejad et al. (2012) reported that coma was the most common clinical feature and all patients had at least some alteration in consciousness. Furthermore, nausea-vomiting and abdominal pain, deterioration of consciousness and dizziness were the most common symptoms in patients intoxicated by drugs in a study of Arlkan et al. (2014).

The highest number of the admitted patients were intoxicated by medicinal drugs $(40 \%)$ followed by agricultural chemicals (35\%) \{organophosphorus $(22.5 \%)$, zic phosphide $(10 \%)$ and hydrogen cyanamide (2.5)\} and carbon mono oxide poisoned cases $(20 \%)$. Similarly, other studies from Turkey and India showed that the major poisons needed ICU admission were medicinal drugs followed by agricultural chemicals (Cengiz et al., 2006; Taghaddosinejad et al., 2012; Manjula et al., 2013). On the other hand, Clark et al. (2011) studied the profile of patients with self-poisoning admitted to ICU in Scotland; they found that the most important agents of acute poisoning were alcohol (41\%), tricyclic antidepressants (28\%), benzodiazepines (21\%), recreational drugs $(23 \%)$, opioids $(14 \%)$, and paracetamol (19\%). The present study revealed that the highest numbers of admitted poisoned cases were organophosphates $(22.5 \%)$ and this could be explained by the nature of El Dakahlia Governorate which is agricultural community of low and middle income with wide use of organophosphorous compounds in agriculture beside its easy availability resulting in accidental and suicidal poisoning.

The length of stay in this study was from 1-25 days in recovered and referral patients and from 1-33 days in died patients. The IQR was from 4 days in recovered and referral patients and 13 days in died patients. Nearly similar, in a study done by Jayakrishnan et al. (2012); patients stayed in the ICU for 1 to 20 days (median-2 days). They attributed longer ICU stay to hypotension upon arrival and the need for mechanical ventilation. While, in UK, Clark et al. (2011) revealed that, Median length of stay in intensive care was 0.7 days and only $12 \%$ of patients remained in intensive care longer than 48 hours as they required ventilatory support, received vasopressors or renal replacement therapy. Hemodynamic instability and disturbed level of consciousness of $47.55 \%$ and $42.5 \%$ of the admitted patients may be reasons for longer stay in ICU in the current study.

In the present study the majority of the admitted patients to ICU were completely recovered $(72.5 \%)$ followed by died cases $(22.5 \%)$. Only two cases $(5 \%)$ that were poisoned by $\mathrm{CO}$ were referred to hyperbaric oxygen therapy units. The results of the present study were partially in accordance with that obtained by Taghaddosinejad et al. (2012); the mortality rate of the poisoned patients in the ICU was $17.7 \%$. Lower mortality rate were reported from studies done in China (3\%), indie (2.8), Scotland (4\%), Alexandria- Egypt $(9.6 \%)$ and Turkey $(0 \%)$ (Lam et al., 2010; Singh, 2011; Clark et al., 2012; Bishr, 2013); Arıkan et al., 2014). The high mortality rate in the present results could be attributed to that patients intoxicated by organophosphates $(22.5 \%)$ and zinc phosphid $(10 \%)$ constituted about one third of the admitted cases to ICU. It is well known that intoxication by agricultural chemicals has a higher mortality rate than medicinal drug (lam, 2010).

The present study revealed statistically significant difference between occupation (unemployment) and patient outcomes. Also, Kelleher et al. (1996) found highly significant correlation between unemployment and self-poisoning. However, Azab (2009) revealed statistically significant relation between sex (male) and patient outcome. In the current study the suicidal attempts represent $52.5 \%$ of the 
studied cases; unemployment may be a leading factor to suicide as it leads to many psychological problems and marital conflicts. In agreement with that Clark et al. (2011) revealed that deliberate self-poisoning is a major health problem worldwide and is a common reason for young adults to be admitted to hospital.

The present study demonstrated a statistically significant difference concerning toxicological amount and frequency. The patients who took the poison repeatedly and in large amount had poor outcome. In contrast, Azab (2009) estimated a statistically significant difference concerning toxicological place as home poisoning had high severity than outside ones. It is well known that the toxic manifestations of a chemical substance may vary depending on both the dose and duration of the exposure. An increase in dose and exposure during the day can cause severe effects and are important factors in patient outcome (Silbergeld, 1998).

The present study showed statistically significant difference between pulse rate, Glasgow coma score, $\mathrm{PH}$ and $\mathrm{PCO}_{2}$ and patients outcomes. This is consistent with Bishr (2013) who revealed a significant relation between disturbed level of consciousness, arterial blood gases and the patient's outcome. Coma (poisoning may induce global cerebral hypoxia directly or secondary to its metabolic effects, shock, and respiratory failure) and loss of brainstem reflexes indicates a poor prognosis (Kirk, 2011). Furthermore, Bansal et al. (2005) reported that bradycardia and tachycardia were associated with bad outcome and mortality compared to normal heart rate. Additionally, lower arterial $\mathrm{pH}$ level on hospital admission may potentially increases the risk of cardiovascular complications in some poisons as TCA poisoning (Yaraghi et al., 2015).

In the present study the relationship between toxicological diagnoses at admission with patient outcomes revealed statistically significant difference regarding zinc phosphid. The recovery and referral percentage of the zinc phosphid was $25 \%$, while the percentage of died cases was $75 \%$. Consistent with this in developing countries like india, Singh et al. (2011) reported that all patients who died had consumed pesticide poison (aluminum phosphide). However, in western countries like USA, Bronstein et al. (2008) found that fatalities occurred most commonly in patients intoxicated by sedative/hypnotics, antipsychotics, opioids, antidepressants, acetaminophen in combination, cardiovascular drugs, and stimulants/street drugs. Zinc and aluminum phosphide are commonly used in Egypt as a rodenticide and as corn preservative as they are easily available and cheap. This high mortality in phosphides could be explained on the fact that they release phosphine gas; a highly toxic gas to the lungs, brain, kidneys, heart, and liver leading to multiorgan failure (through blocking cytochrome c oxidase and free radical generation) (Kariman et al., 2012).

The decision to admit a patient with a toxic exposure to an ICU should be based upon clinical criteria that relate to the stability of the airway, respiratory system and cardiovascular system. disturbed level of consciousness which may need endotracheal intubation, presence of post ingestion seizure, systolic blood pressure $<80 \mathrm{mmHg}$, non sinus cardiac rhythm, second and third degree atrioventricular block, QRS >12 milliseconds, partial pressure of arterial carbon dioxide $\left(\mathrm{Paco}_{2}\right)>45 \mathrm{mmHg}$, in addition to the requirement for ICU interventions (intubation, vasopressor, antiarrhythmic, dialysis or hem filtration) are the main indicators to ICU admission (Kirk, 2011).

In the present study, the major ICU admission criteria were hemodynamic instability $(47.5 \%)$ or altered mental status $(42.5 \%)$ or combined hemodynamic instability and altered mental status $(35 \%)$. While, the admission criteria in a study done by Azab (2009) were both hemodynamic instability and altered mental status $(61.1 \%)$ followed by altered mental status $(16.3 \%)$, then hemodynamic instability $(14.5 \%)$ while those presenting with the three admission criteria were $(8.1 \%)$. Moreover, altered mental status $(65.3 \%)$, aspiration pneumonia $(11.7 \%)$, rhabdomyolysis $(10.6 \%)$, adverse cardiac events $(5.7 \%)$, shock $(7.9 \%)$, seizure $(8.7 \%)$ and acute renal failure $(3.7 \%)$ were the main complications of poisoning that need ICU admission (Lam et al., 2010).

Although many of the patients' characteristics were significantly associated with patient outcome, yet none of them considered significant predictor for death. So, the suggested scoring system depended mainly on clinical data and simple rapid investigation that was supposed to be associated with patient outcome and prognosis (Blood pressure, pulse rate, respiratory rate, Glasgow coma score, convulsions, $\mathrm{PH}, \mathrm{PO}_{2}, \mathrm{PO}_{2}$, $\mathrm{HCo}_{3}, \mathrm{SO}_{2} \%, \mathrm{Na}$ and $\mathrm{K}$ ). Smith and Nielsen (1999) revealed that admission to intensive care unit is indicated in patients with potential or established failure of one or more organ with expected recovery. The need for diagnosis and treatment of organ dysfunction with high risk of complications are another indicator. Furthermore, early admission will decrease the number \& extent of organ impairment and length of patient stay in ICU. According to the best of our knowledge, this is the first study to report four prognostic grades for poisoned cases admitted to intensive care unit. Moreover, we have established a rapid bedside although detailed clinical scoring system. The suggested scoring system that we had constructed depends mainly on clinical data and simple rapid investigation to make it rapid and simple in centers with low equipments. We hope that it may be helpful for rapid assessment of the patients after further validation.

The present study limitations were small number of patients included and short duration of the study as well as analytical confirmation of poisoning was not done to all patients and diagnosis depended mainly on the history and clinical examination. Additionally, it was conducted in a single center equipped with moderate facilities located in a governorate capital; hence, the results cannot be generalized. 


\section{From the result of the present study, it can be concluded that:}

Acute poisoning is one of the most important causes of emergency hospital and ICU admissions and mortalities. The highest numbers of admitted poisoned cases were from drugs and agricultural chemicals taken mainly orally at home in suicidal attempts, raising the attention about the danger of household products in poisoning and suicide. Treatment protocols require updating to reserve decontamination for early presenting patients. $72.5 \%$ of cases that were admitted to the ICU were discharged, and $22.5 \%$ of cases died mainly due to organophosphorus and phosphide reflecting the need for improving the ICU facilities and awareness programs about pesticides toxicity. We hope that this study can increase the awareness about the profile of the intoxicated patients and the prediction of their outcomes. We recommend the presence of a channel of communication between the general care centers and the poison control centers for consultation about the protocol of treatment of poisoned patients. Nationwide, a mechanism of communication between the poison centers must be done to benefit from the different expertise and to make a uniform protocol for treatment of poisoned cases to decrease the mortality rate and the burden on hospitals resources.

\section{References}

Arıkan M, Temel V, Acat M et al., (2014): Incidence of intoxication in Karabuk (Turkey) in 2013. Br J Med Med Res. 4(18): 3406-3414.

Azab RM (2009): Role of intensive care unit (ICU) in acute poisoning cases admitted to Menoufiya University Hospitals over one year (2008). (M.B,B.ch) Thesis at Faculty of Medicine, Menoufiya University.

Bansal A, Singhi SC, Singhi PD et al., (2005): Non traumatic coma. Indian J Pediatr. 72 (6): 467473.

Bishr AA (2013): Criteria for intensive care unit admission among acute intoxicated patients admitted to Alexandria Main University Hospital. M.S.C. Thesis in Forensic Medicine and Clinical Toxicology Department, Faculty of Medicine, Alexandria University.

Bronstein, AM.D, Spyker DA, Cantilena LR et al., 2008): 2007Annual report of the american association of poison control centers' National Poison Data System (NPDS): 25th Annual Report. Clin Toxicol. 46:927-1057.

Caldeira VMH, Júnio JMS, de Oliveir AMRR et al., (2010): Criteria for patient admission to an intensive care unit and related mortality rates. Rev Assoc Med Bras. 56(5): 528-534.

Cengiz M, Baysal Z, Ganidagli S (2006): Characteristics of poisoning cases in adult intensive care unit in Sanliurfa, Turkey. Saudi Med J. 27: 497-502.

Chiu LQ, Lim BL, Vasu A (2011): Poison exposure in the emergency department: a Singaporean experience Hong Kong. j. emerg. med. 18(4): 197-203.

Chyka PA, Seger D, Krenzelok EP et al, (2005): Position paper: Single-dose activated charcoal. Clin Toxicol. 43(2):61-87.
Clark, D Murray, DB Ray D (2011): Epidemiology and outcomes of patients admitted to critical care after self poisoning. JICS. 12:267-273.

Clark R (2004): The practice of medical toxicology. In Dart Medical Toxicology. Dart RC (ed). 3rd ed. Lippincott Williams \&Wilkins: Philadelphia; 36.

Duger C, Ozdemir K I, Ak K et al., (2013): Analysis of individual and clinical factors in acute poisoning cases in the intensive care unit. Basic Clin Sci. 2: 101-107.

Jayakrishnan B, Al Asmi A, Al Qassabi A et al., (2012): Acute drug overdose: Clinical profile, etiologic spectrum and determinants of duration of intensive medical treatment. Oman Med J. 27(6): 501-504.

Kariman H, Heydari K, Fakhri M (2012): Aluminium phosphide poisoning and oxidative stress: serum biomarker assessment. J Med Toxicol. 8(3):281284.

Kavalci C, Demir A, Arslan ED et al., (2012): Adult poisoning cases in Ankara: Capital city of Turkey. IJCM. 3(7): 736-739.

Kelleher MJ, Kelleher MJ, Daly M (1996): Deliberate self-poisoning, unemployment and public health. Suicide Life Threat Behav. 26(4):365-373.

Kirk MA (2011): Use of the intensive care unit. In: Goldfrank's Toxicologic Emergencies. Flomenbaum NE, Goldfrank L R, Hoffman R S, Howland M A et al.,(eds). $9^{\text {th }}$ Ed. McGraw-Hill: New York; 148-153.

Lam SM, Lau ACW, Yan WW (2010): Over 8 years experience on severe acute poisoning requiring intensive care in Hong Kong, China. Hum Exp Toxicol. 29:757-765.

Liisanantti J (2012): Acute drug poisoning: outcome and factors affecting outcome. Acta Univ. Oul. D 1154:1-67.

Manjula M, Sanjeev S, Shruthi G (2013): A study of pattern and outcome of acute poisoning cases in a tertiary care hospital. JEMDS. 27: 4877-4882.

Silbergeld EK (1998): Toxicology. Encyclopedia of occupational health and safety. $4^{\text {th }}$ edition. Chapter 33.

Singh O, Javeri y, Juneja D (2011): Profile and outcome of patients with acute toxicity admitted in intensive care unit: Experiences from a major corporate hospital in urban India. Indian $\mathbf{J}$ Anaesth.55 (4): 370-374.

Smith $G$ and Nielsen M (1999): Criteria for admission. BMJ. 5; 318(7197): 1544-1547.

Taghaddosinejad F, Sheikhazadi A, Yaghmaei A et al., (2012): Epidemiology and treatment of severe poisoning in the intensive care unit: Lessons from a one-year prospective observational study. J Clinic Toxicol S1:007. doi:10.4172/21610495.S1-007.

Takrouri, M S M (2004): Intensive care unit. IJH, 3(2): DOI: $10.5580 / 1 \mathrm{c} 97$.

Vale JA and Kulig K (2004): American academy of clinical toxicology, European association of poisons centres and clinical position paper: 
Gastric lavage. $J$ Toxicol Clin Toxicol. 42(7):933-943.

Yaraghi A, Eizadi-Mood N, Katani M et al., (2015): Clinical study: Arterial blood gas analysis and the outcome of treatment in tricyclic antidepressants poisoned patients with benzodiazepine coingestion. Anesthesiol Res Pract. 2015, Article ID 232401, 5 pages http://dx.doi.org/10.1155/2015/232401.

\section{الملخص العربى}

\section{التنبؤ بمصيرمرضى التسمم الحاد الذين أدخلوا لوحدة العناية المركزة في المنصورة ـ محافظة الدقهليةـ مصر أ.د.م مرفت عريبى و أ.د عائشة مقلد ا د. نهال شهاب بو ط. هلى قاسمب}

التسمم الحاد هو سبب شائع للقبول في أقسام الطوارئ و وحدات العناية المركزة، كما أنه يعتبر حالة طوارئ طبية هامة تتطلب التشخيص المبكر والبدء السريع في العلاج. كان الهدف من هذه الدراسة هو تقييم خصائص مرضى التسمم الحاد الذين تم ادخالهم وحـدة العناية المركزة و التنبؤ بنتائجهم و أيضا تصميم نظام حـرزي بشأن أولوية القبول بالعناية المركزة. أجريت هذه الدراسة على ·ـ عريضا تم ادخالهم الى وحدة العناية المركزة في مستشفى المنصورة العام - مصر خلال فترة 7 أشهر. و قد سجل التاريخ الشخصى و الطبى و السمى والفحص الأكلينكى والأبحاث الروتينية

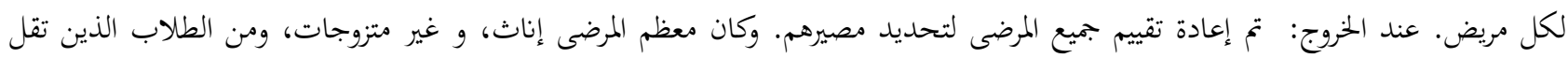

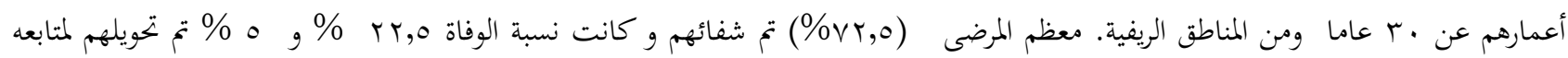
العلاج بوحدة العلاج بالأكسجين تحت الضغط بعد تسممهم بغاز اول اوكسيد الكربون. حضر العديد من المرضى بضيق فى حدقة العين (0ءم) وكسل الجهاز التنفسي و الغيبوبة(42.5\%) وزيادة دقات القلب (47.5\%)، والغثيان والقيء (75\%) وكان أكبر عدد من الحالات قد تسمم بالعقاقير الطبية (·ع ٪) تليها المواد الكيميائية الزراعية (هץ\%). وقد لوحظ فرقا يعتد به إحصائيا بين الوظيفة وكمية و تكرارالسم ، معسدل النبض، مقياس غـلاسكو للغيبوبة، الأس الهيدروجيني و مصير المرضى. على الرغم من أن العديد من خصائص المرضى ارتبطت بشكل كبير مع نتائج المرضى إلا أن أيا منهم لا يعتبركمتبئى هام للموت. لذلك، يعتمد نظام التسجيل المقترح أساسا على المعطيات السريرية والأبحاث الروتينية السريعة البسيطة التي قد تكون مفيدة للتقييم السريع للمرضى بعد إجراء مزيد من التحقق من صلاحيتة . يوصى بوجود وسيلة للاتصال بين مراكز الرعاية العامة و مراكز السموم للتشاور حول بروتكول علاج حالات التسمم.كما يجبب عمل ألية للتواصل بين مراكز السموم على مستوى الجمهورية للاستفادة من الخبرات للعمل ببروتكول موحد لعلاج حالات السموم المختلفة لتقليل نسبة الوفيات و تقليل العبء على موارد 